\title{
THE PLANTS OF THE TREVALLYN STATE RECREATION AREA, TASMANIA
}

\author{
by A.V. Ratkowsky, D.A. Ratkowsky and G. Kantvilas
}

(with one table and one text-figure)

\begin{abstract}
Rutkowsky, A.V., Ratkowsky, D.A. \& Kuntvilas, G., 1993 (31:viii): The plants of the Trevallyn State Recreation Area. Pap. Proc. R. Soc. Tasm. 127: 49-54. https://doi.org/10.26749/rstpp.127.49 ISSN 0080-4703. PO Box 46, Kings Meadows, Tasmania 7249 (AVR, DAR); The Tasmanian Herbarium, GPO Box 252C, Hobart, Tasmania 7001 (GK).
\end{abstract}

A census of species of all plant groups, cxcluding free-growing fungi and algae, of the Trevallyn State Recreation Area near Launceston, Tasmania, is presented. The list of 232 species of flowering plants includes 15 species that are either rare or considered to be vulnerable, or are unreserved or poorly represented in state reserves. Although gazetted as a state recreation area, the management policy for the reserve is consistent with the preservation of existing native vegctarion.

Key Words: Trevallyn State Recreation Area, vascular plants, bryophytes, lichens, Northern Tasmania, rare species, threatened species.

\section{INTRODUCTION}

The Trevallyn State Recreation Area (SRA) lies within $5 \mathrm{~km}$ of the centre of the City of Launceston and is the largest reserve near to Tasmania's second largest city, with an arca of c. 400 ha. The SRA (fig. 1) is bounded on its western side by Trevallyn Lake, formed by the Trevallyn Dam on the South Esk River. Bclow the dam, the river flows southeastwards, turning northeastwards at the extreme southern tip of the SRA, ultimatcly forming the River Tamar at its junction with the North Esk River outside the boundaries of the Reserve. Private property and Reatta Road form part of the northern boundary of the SRA, with the northeastern boundary abutting on houses in the suburb of Trevallyn. At its extreme eastern side, the SRA shares a border with the Cataract Gorge Rescrve.

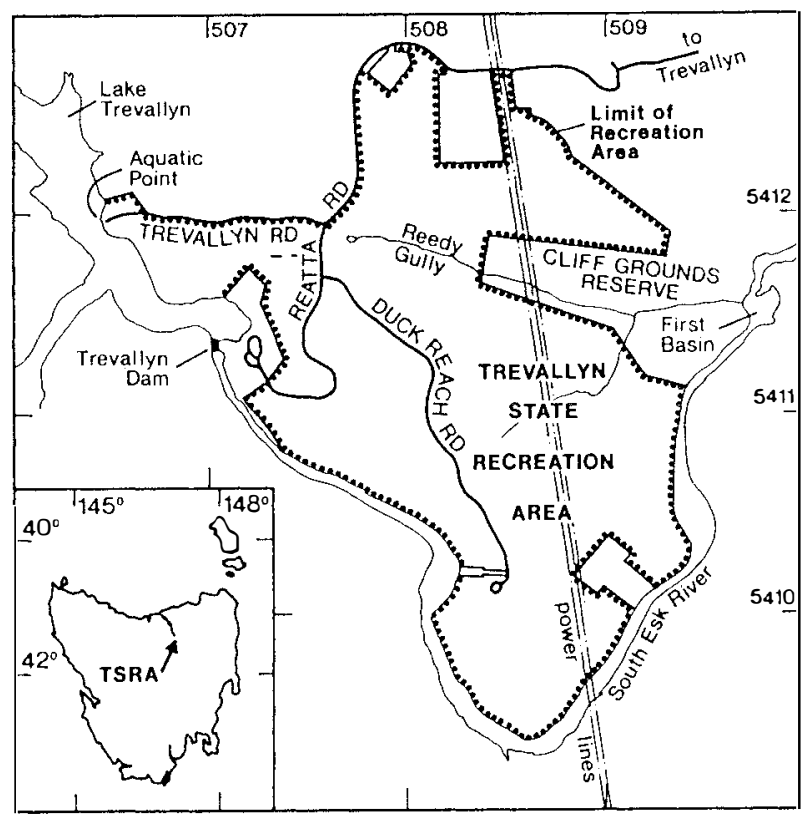

FIG. 1 - Location map for Trevallyn State Recreation Area.
The SRA has a bedrock of Jurassic dolerite. The soils on this have a topsoil that is mostly a brown or grcy-brown loam, with a grey, sandy loam subsurface which changes sharply at about $0.3 \mathrm{~m}$ depth to a yellow-brown clay ('Tasmanian Department of Agriculturc 1981). Minor occurrences of lateritic soils are found throughout the area (see, for example, Longman 1964, 1966: 21). The soil depth is extremely variable, bcing very shallow and stony in some places where rock is exposed at the surfacc. Gencrally the soils are well drained, although they can become waterlogged in arcas where there is a heavy clay subsoil and little slope (Tasmanian Department of Agriculture 1981).

The elevation of the SRA ranges from c. $25 \mathrm{~m}$ to c. $225 \mathrm{~m}$, the terrain undulating considerably throughout the arca and presenting a varicty of aspects. The area is bordered on its southwestern and southeastern sides by the gorge of the South Lsk River, a gorge cut by a stream cnlarged by diversion into the drainage basin of the ancestral South Esk by a basalt flow near Evandalc (Nicolls 1960).

Prior to 3 June 1980, the date the Trevallyn SRA was proclaimed, much of the arca had been used as a farm. The management of the reserve, now carricd out by the Parks and Wildlife Service, a division of the Department of Environment and Land Management, pursues a policy of allowing regrowth of native vegetation. However, controlled burning of some areas is undertaken for firc hazard reduction purposes because the rescrve is situated on the outskirts of a major urban arca. Also, during a scvere drought during the mid-80s, cattle were agisted there intermittently.

\section{SURVEY ZONES AND METHODS}

For the botanical survey, the area of 400 ha constituting the Trevallyn SRA was divided into four zones to reflect fundamental differences in the various vegetation types present (fig. 1). By far the most extensive vegetation formation in the reserve is dry sclerophyll forcst; its predominant large woody species include Acacia dealbata, Eucalyptus amygdalina and E. viminalis, with conspicuous quantities of Lomandra longifolia and Pteridium esculentum also present as an understorey. A sccond vegetation type, which may be described as riparian, is found along the gorge of the South Esk River. In addition to having certain common large specics such as Cassinia aculeata and Beyeria viscosa which were less frequently 
represented in the dry sclerophyll zone, this zone contains a locally abundant stand of Prostanthera rotundifolia, a species known only from a few localities in the north and east of Tasmania, and considered to be unreserved and vulnerable (Kirkpatrick et al. 1991). Also present in this zone is Micrantheum hexandrum, whose occurrence in Tasmania is exclusively northern and eastern (Orchard 1991). A third environment, which contains several fern species (of the Blechnaceae and Dicksoniaceae) not found elsewhere in the reserve, borders a perennial or intermittent creek (Reedy Gully, fig. 1). The fourth zone recognised in this survey is an area cleared of most trees and shrubs underneath the electricity lines that form part of the Hydro-Electric Commission's grid, which includes the Trevallyn Power Station on the Tamar River. This zone contains a large variety of herbaceous species.

Visits to the survey zones were made or a weekly or twiceweekly basis between September 1991 and May 1992. An attempt was made to cover as much of the area as possible, using all of the available tracks and also travelling crosscountry wherever the terrain permitted. Species in planted beds along roadsides, landscaped picnic areas and a rubbish tip opposite the commercial Cable Hang Glider installation were omitted from the survey. Aside from these, the survey includes all other introduced species.

\section{RESULTS}

Table 1 lists the plant species recorded; it includes 232 species of flowering plants (of which 161 are native to Tasmania, including five endemics), ten fern species, 39 mosses, 18 liverworts and 57 lichens. For the flowering plants and ferns, a measure of abundance (local, rare, occasional, ftequent or common) is given for each species in each zone. For the mosses and liverworts, no measure of abundance is given, because positive identification of a species in the field is difficult, but table 1 records the zones in which each species was found. For the lichens, which often require chemical analysis for determination, only the presence of species is recorded. The scientific names of the flowering plants and ferns follow Buchanan et al. (1989); those of the mosses follow Dalton et al. (1991); those of the liverworts follow Ratkowsky (1987). For the lichens, scientific names and authorities aregiven in full, as there have been many alterations and additions to the checklist previously published by Kantvilas (1989).

\section{DISCUSSION}

Among the flowering plants, 15 species are considered to be of interest because of their rareness, vulnerability or unreserved status. Kirkpatrick et al. (1991) classified all of Tasmania's native higher plants as to the extent to which they were present in national parks or "equivalent reserves", categories which do not include state recreation areas. Three species, Brunonia australis, Goodia pubescens and Prostanthera rotundifolia, are unreserved anywhere in Tasmania, and a fourth species, Centipeda cunninghamii, is reserved only at Lavinia Nature Reserve on King Islánd. A fifth species, Myriophyllum integrifolium, is reserved only at Epping Forest Nature Reserve and is considered to be vulnerable. The remaining ten species, Hydrocotyle callicarpa, Cynoglossum australe, Myosotis australis, Crassula decumbens, Scutellaria humilis, Persicaria prostrata, Aphelia gracilis, A. pumilio, Carex longebrachiata and Dichopogon strictusare accorded the status "r2" by Kirkpatrick et al. (1991), which means that they are known to occur in 20 or less $10 \mathrm{~km} \times 10 \mathrm{~km}$ National Mapping squares in Tasmania. Except for Crassula decumbens, which is known from five national parks or equivalent reserves, the other nine species are recorded from only one reserve each.

The most diverse families of flowering plants were the grasses (Poaceae) with 30 species (18 native), the daisies (Asteraceae) with 29 species (18 native), the wattles and "pea" flowers (Fabaceae) with 18 species (nine native), and the sedges (Cyperaceae) with 15 species (14 native). Only four orchid species were recorded.

None of the cryptogams was considered to be particularly noteworthy or unusual, with the possible exception of the lichen record reported here as Bacidia cf. millegrana, which requires comparison with type material to confirm its identity. Most of the species of moss and liverwort grew in moist or shady areas within dry sclerophyll environments. A few ate adapted to the very dry conditions that ptevail during the summer months, being capable of withstanding desiccation but returning to a healthy appearance once the autumn and winter rains bring moister conditions. The lichens wete mainly collected from the bark of trees, with several growing on soil, rocks and stones at ground level. Most species are typical of dry sclerophyll forests in Tasmania. The most common species on trees are the foliose lichens, Parmelina conlabrosa, P. pseudorelicina and Flavoparmelia rutidota, and the tiny crustose species Buellia disciformis. On soil, Heterodea muelleri, Cladia aggregata and species of Cladonia predominate, whilst species of Xanthoparmelia are common on rocks. As with the bryophytes, lichens were often found growing in moister sites within the dry sclerophyll environment. Hence, the conditions that determine where these species occur are related more closely to the microclimate than to the broad-scale vegetation classification, such as dry sclerophyll, gorge, etc., used here.

Since much of the survey area had been used as a farm prior to 1980, the current composition of the vegetation is undoubtedly vastly different from what it was prior to European settlement. The present management policy of the Parks and Wildlife Service is for minimal interference, allowing for natural regrowth of the vegetation. Nevertheless, because of the reserve's location on the outskirts of Tasmania's second largest urban area, the Service also has the responsibility to reduce fire hazards by occasional burning for fuel reduction. It is possible, however, that increasing wildlife populations will lessen the need for hazard reduction burning to some extent in the future. Another unpredictable factor is the possibility of future periods of severe drought, resulting in a shortage of feed for farm animals. Should that happen, it is possible that the Tasmanian Government may require that the reserve be used for cattle agistment, as it did in the mid-80s. Consequently, it is not possible to guarantee that the vegetation will be allowed to achieve a "climax community" in any sense, nor predict that the diversity of plant species in the reserve will increase in the future.

\section{ACKNOWLEDGEMENTS}

The authors are grateful for the assistance in plant identification provided by Mary Cameron (Queen Victoria Museum and Art Gallery, Launceston), Alex Buchanan and 
Dennis Morris (both of the Tasmanian Herbarium, Tasmanian Museum and Art Gallery, Hobart). Rex Gatenby, Parks and Wildlife Service, provided information on the history of the Trevallyn State Recreation Area and about current management practices. Kathy Noble, Richard Doyle and Greg Pinkard, all of the Mount Pleasant Laboratories, Department of Primary Industry and Fisheries, directed the authors' attention to information on the soils, geology and geomorphology of the SRA. Ross Corkrey prepared the figure.

\section{REFERENCES}

Buchanan, A.M., McGeary-Brown, A. \& OrChard, A.E., 1989: A CENSUS OF THE VASCULAR PLANTS OF TASMANIA. Occ. Publ. Tasm. Herb. 2. Tasmanian Museum and Art Gallery, Hobart.

Daiton, P.J., Seppeit, R.D. \& BuChanan, A.M., 1991: An annotated checklist of Tasmanian mosses. In Banks, M.R., Smith, S.J., Orchard, A.E. \& Kantvilas, G. (Eds): ASPECTS OF TASMANIAN BOTANY - A TRIBUTE TO WINIFRED CURTIS. Royal Society of Tasmania, Hobart: 15-32.
Kantvilas, G., 1989: A checklist of Tasmanian lichens. Pap. Proc. R. Soc. Tasm. 123: 67-85.

Kirkpatrick, J., Gilfedder, L., Hickie, J. \& Harris, S., 1991: Reservation and conservation status of Tasmanian higher plants. Sci. Rep. Wildl. Div. 91/2. Dep. Parks, Wildl. \& Herit., Tasm.

Longmas, M., 1964: Launceston, Tasmania. Tasm. Dep. Mines 1 Mile Geol. Atlas Ser., Sheet 39 (8315S).

Longman, M., 1966: Launceston, Tasmania. Tasm. Dep. Mines 1 Mile Geol. Atlas Ser., Expl. Rep., Sheet 39 (8315S).

Nicolls, K.D., 1960: Erosion surfaces, river terraces, and river capture in the Launceston Tertiary Basin. Pap. Proc. R. Soc. Tasm. 94: 1-12.

OrChaRd, A.E., 1991: A new species of Micrantheum (Euphorbiaceae) from Tasmania. In Banks, M.R., Smith, S.J., Orchard, A.E. \& Kantvilas, G. (Eds): ASPECTS OF TASMANIAN BOTANY - A TRIBUTE TO WINIFRED CURTIS. Royal Society of Tasmania, Hobart: 59-64.

RatKowsky, D.A., 1987: Check-list of the Tasmanian liverworts. Pap. Proc. R. Soc. Tasm. 121: 153-158.

Tasmanian Department of Agriculture, 1981: GARDEN GUIDE, SOILS OF LAUNCESTON. G61(a)/81.

(accepted 6 May 1993)

\title{
TABLE 1 \\ List of species found in Trevallyn SRA
}

\begin{abstract}
Zones: D, dry sclerophyll; G, slopes of the gorge of the South Esk River; C, Reedy Gully Creek and environs; P, areas cleared underneath electricity power lines.

Abundances: 1, local (growing in a few places only, sometimes abundantly); r, only 1-3 plants observed, rare; o, widespread but only occasional; $f$, widespread and frequent; $c$, widespread and common.
\end{abstract}

(I) following a species name indicates that this species has been introduced to Tasmania. All other species are considered to be native. (E) indicates species endemic to Tasmania.

\begin{tabular}{cc}
\hline Species & Zones \\
& D G C P
\end{tabular}

Species Zones

\section{DICOTYLEDONES}

\section{APIACEAE}

Conium maculatum (Hemlock) (I)

Daucus glochidiatus (Austral Carrot)

Hydrocotyle callicarpa (Pennywort)

Hydrococyle hirta (Hairy Pennywort)

ASTERACEAE

Arctotheca calendula (Cape Weed) (I)

Bedfordia salicina (Blanket Leaf) (E)

Brachyscome sp. (Field Daisy)

Carduus tenuiflorus (Winged Slender Thistle) (I)

Cassinia aculeata (Dolly Bush)

Centipeda cunninghamii (Sneezeweed)

Centipeda minima (Sneezeweed)

Chrysanthemoides monilifera (Boneseed) (I)

Cirsium vulgare (Spear Thistle) (I)

Cotula australis (Common Cotula)

Cotula reptans (Creeping Cotula)

Filago gallica (French Filago) (I)

Hypochoeris glabra (Smooth Cat's Ear) (I)

Hypochoeris radicata (Flat-weed; Cat's Ear) (I)

Lagenifera stipitata (Blue Bottle-Daisy)

Leontodon taraxacoides (Hawkbit) (I)

Leptorbynchos squamatus (Scaly Buttons)

Microseris lanceolata (Native Dandelion)

Olearia stellulata (Daisy Bush)

Pseudognaphalium luteo-album (Cudweed)

Senecio glomeratus (Fireweed)

Senecio hispidulus (Scabrid Fireweed)

Senecio jacobea (Ragwort) (I)

Senecio linearifolius (Fireweed)

Senecio minimus (Fireweed)

Senecio quadridentatus (Cotton Fireweed)

Solenogyne gunnii (Solenogyne)

Sonchus asper (Prickly Sow Thistle) (I)

Taraxacum officinale (Dandelion) (I)

BORAGINACEAE

Cynoglossum australe (Hound's-Tongue)

Cynoglossum suaveolens (Hound's-Tongue)

Lithospermum officinale (Gromwell) (I)

Myosotis australis (Forget-me-not)

Myosotis discolor (Forget-me-not) (I)

Myosotis sylvatica (Forget-me-not) (I)

BRASSICACEAE
o o f Cardamine hirsuta (Bittercress) (I)

Rorippa dictyosperma (Bittercress)

BRUNONIACEAE

$\mathrm{r}$ Brunonia australis(Blue Pincushion)

CAMPANULACEAE

Pratia pedunculata (Matted Pratia)

Wablenbergia gymnoclada (Bluebell)

Wablenbergia stricta (Bluebell)

CAPRIFOLIACEAE

Sambucus gaudichaudiana (Native Elder) I r

CARYOPHYLLACEAE 
Table I cont.

\begin{tabular}{cc}
\hline Species & \%ones \\
& D C C P P \\
\hline
\end{tabular}

\section{CASUARINACEAI:}

Allocasuarina verticillata (She-()ak)

CONVOL.VUL.ACEAl:

Dichondra repens (Kidney-wced)

CRASSUIACEAI:

Crassula decumbens (Spreading Crassula)

Crassula peduncularis (Purple Crassula)

DILLIENIACI:Al:

llibberial birsuta (Guineal lilower) (E)

llibbertia riparia (Guinca lilower)

DIPSACAl:Al:

Dipsacus sylvestris (Teasel) (I)

DROSERACENI:

Drosera peltatu ssp. peltatra (Sundew)

Drosera peltata ssp. peltata var. gracilis (Sundew)

Drosern lygmaen (Pigny Sundew)

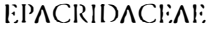

Acrotriche serrulata (Ant's Delight)

Astroloma humifissum (Narive Cranberry)

l:pacris impressit (Common Heath)

l.issamthe strigosa (Peach Berry)

ERICALAl:

lirica Lusitunica (Spanish Heath) (I)

EUPHORBIACEAI:

Beyerin viscosal (Pinkwood)

Micruntheum hexandrum (Box Micrancheum)

P'oranthera microphylla (Small-leaf Poranthera)

F $\triangle B \Lambda C E \Lambda \mathrm{E}$

Acacia dealbata (Silver Wattle)

Acacia mearnsii (Black Wattle)

Acacia melanoxylon (Blackwood)

Acacia mucronata (Narrow-leaved Wartle)

Bossinen prostrata (Creeping Bossiacia)

Cytisus scoparius (Broom) (I)

Daniesia latifolia (Bitter I.c.af)

Gienista monspessulanat (Canary Broom) (I)

Goodia pubescens (Silky Cloverrree)

l.ouea sp. (Hovea)

Incligofera australis (Native Indigo)

Lotus peclunculatus (Circater Trefoil) (I)

Lotus subbiflorus (Hairy Bird's-foot Trefoil) (I)

l.otus tenuis (Slender Bird's-foot Trefoil) (I)

Trifolium elubium (Yellow Suckling Clover) (I)

Trifolium repens (White Clover, Dutch Clover) (I)

Trifolium subterraneum (Subtersancan Clover) ( I)

(Nlex entropaens (Gorse, Furze) (I)

I:UMARIACEAE

Fumaria muralis (Fumitory) (I)

GIENTIANACLAL:

Centaurium erytluraea (Common Centaury),(I)

Cicendia filiformis (Slender Cicendia) (I)

Scbuten ovata (Ycllow Scbaci)

CIERANIACEAE

Lirodium moschatum (Musk Storksbill) (I)

Ceranium potentilloieles (Gicranium)

Pelargonium inodorum (Pelargonium, "Cicranium")

GOODENIACENI:

Goodenia elongata (Lanky Coodenia)

Goodenia lanata (Narive Primrose)

Goodenia ovata (Parrot's Food)

HAI.ORAGAC.EAL:

Gonocarpus tetragynus (Common Raspwort)

Myrioplsyllum integrifolium (Water Milfoil)

I AMI $\Lambda$ Cl:Al:

Mentha pulegium (Pennyroyal) (I)

Prostanthera rotundifolia (Round-lealf Mint-bush)

Prunella vulgaris (Self-heal) ( I)

Scutellaria humilis (Dwarf Skullcap)

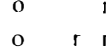

c o
Species

\%ones

D ( ; C P

I.AURACEAI:

Carsytba melantha (Native Dodder)

I.INACl:Al:

$f$ of o Linum trigynum (lirench lilax) (I)

MYRTACEAE:

$\begin{array}{llll}1 & 1 & f & \text { Calytrix tetragona (Fringe Myrte) } \\ \text { o } & \text { o } & \text { litucalyptus amygdalina (Black Peppermint) (E) }\end{array}$

Inucalyptus ovata (Swamp Cium)

I:ucalyptus viminalis (White Gum, Manna Gum)

leptospermum lanigerum (Woolly Teit-trec)

OLLACEAL:

Norelaca ligustrina (Native Olive)

ONAGRACEAE:

E:pilobium billardieriunum ssp. cinereum (Willowherb)

Epilobium gunnianum (Willowherb)

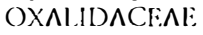

Oxillis corniculata sens. lat. (Yellow Wood Sorrel)

PASSIFI.ORACEAL:

Passiflora cinnabarina (Passion-flower) (I)

PI'I"IOSPORАCEAE:

Billardiera scandens ( $\Lambda$ pple Berry)

Bursaria spinosa ( $\Lambda$ uscralian Prickly Box)

Pittosportum bicolor (Chcesewood, Tallow-wood)

PI ANTACINACEAl:

o c f Plintago coronopus (Buck's-horn Plantain) (I)

Plantago lanceolata (Ribwort) (I)

Plantago varia (Variable Plantain)

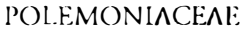

Nanarretia squarrosa (Cilifornian Stinkweed) (I)

POI.YCIALACEAL:

Comesperma volubile (Blue Love Creeper)

POLYGONAClNA:

Persicaria prostrata (Creeping Knotweed)

Rumex acetosella (Shecp's Sorrel) (I)

Rumex obtusifolius (Broad-leaf Dock) (I)

PRIMULACEAE:

Anagrtlis arvensis ssp. arvensis (Pimpernel) (I)

RANUNCUIACIAE:

Clematis aristata (Australian Clemaris)

Ranunculus lappaceus (Butrercup)

RIHAMNACEAL

Po!naderris apetala (Dogwood, Native Hazel)

ROSACENI:

Acatena echinata (Shecp's Burr)

Aca'na novae-zelaneliate (Buzz.y, Biddy-widdy)

(rrataegus monos'na (l lawthorn) (I)

Rosa canina (I)og Rose) (I)

Rulus fruticosus (Blackberry) (I)

Ruhus parvifolius (Native Raspberry)

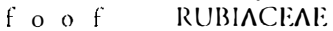

Aijerula conferta var. conferta (Common Woodruff)

o $\quad$ o $\quad$ c

111

1

c c c o

c c c o

f

fo

c c c

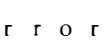

$r \quad r \quad r$

f c c o

r I

f

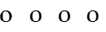

o

1

o $\quad$ o r

o $\quad$ o $f_{\text {r }}$

o o

f c c

$f$ if $f$

$f \subset c f$

r r

o 1

$f \circ f f$

Coprosma quatlrifida (Native Currant)

Galium aparine (Cleavers, Coosegrass) (I)

Galium australe ('langled Bedstraw)

(ialium gauclichaudii (Rough Bedstraw)

(ialium nurrale (Small Bedstraw) (I)

Opercularin ounta (Broad-lcaf Stinkweed)

Sherarelia arvensis (liicld Madder) (I)

RUTACEAE

Correa laurenciana (Native Fuchsia)

Correa reflexa (Native l:uchsia)

$\begin{array}{lll} & \mathbf{r} \\ 0 & 0\end{array}$

$\begin{array}{llll}0 & 0 & & 0 \\ 0 & 0 & 0 & 0\end{array}$

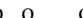

$r$

r r

1

r o

SAI ICACFAl:

Salix "fragilis"(Crack Willow) (I)

f

SANTAIACLAL:

Liocarpos cupressiformis (Native Cherry)

SAIINDACEAL:

Dorlonaea viscosa (Native Hop)

SCROPHUIARIACFAE.

Girtiola latifolia (Broad-leaf Brooklime) $f f f o$

o $f$

111 
Table 1 cont.

\begin{tabular}{cc}
\hline Species & Zones \\
& D G C P
\end{tabular}

Parentucellia latifolia (Common Barrsia) (I)

Parentucellia viscosa (Sticky Barrsia) (I)

Verbascum virgatum (Twiggy Mullein) (I)

Veronica calycina (Speedwell)

Veronica gracilis (Speedwell)

STACKHOUSIACEAE

Stackhousia monogyna (Narive Mignonette, Candles)

STYLIDIACEAE

Stylidium graminifolium (Trigger Plant)

UR'TICACEAE

Urtica incisa (Nettle)

VIOLACEAE

Hymenanthera dentata (Tree Violet)

Viola hederacea (Ivy-leaf Violet)

\section{MONOCOTYLEDONES}

\section{CENTROLEPIDACEAE}

Aphelia gracilis (Slender Aphelia)

Aphelia pumilio (Dwarf Aphelia)

Centrolepis aristata (Pointed Centrolepis)

Centrolepis strigosa (Hairy Centrolepis)

\section{CYPERACEAE}

Carex appressa (Tall Sedge)

Carex gaudichaudiana (Sedge)

Carex inversa (Sedge)

Carex longebrachiata (Drooping Sedge)

Cyperus eragrostis (Umbrella Sedge) (I)

Cyperus tenellus (Tiny Flat-sedge)

Eleocharis acuta (Common Spike-rush)

Eleocharis sphacelata (Tall Spike-rush)

Isolepis fluitans (Floating Club-rush)

Isolepis platycarpa (Club-rush)

Lepidosperma elatius var. ensiforme (Tall Sword-sedge)

Lepidosperma inops (Little Sword-sedge) (E)

Lepidosperma laterale (Variable Sword-sedge)

Lepidosperma lineare (Narrow Sword-sedge)

Schoenus apogon (Common Bog-rush)

HYPOXIDACEAE

Hypoxis glabella (Yellow Star)

Hypoxis hygrometrica (Golden Weather-glass)

IRIDACEAE

Diplarrhena moraea (White Iris)

Gladiolus undulatus (Wild Gladiolus) (I)

JUNCACEAE

Juncus articulatus (Jointed Rush) (I)

Juncus bufonius (Toad Rush)

Juncus pallidus (Pale Rush)

Juncus procerus (Robust Rush)

Juncus subsecundus (Finger Rush)

Luzula densiflora (Woodrush)

\section{LILIACEAE}

Bulbine bulbosa (Bulbine Lily)

Burchardia umbellata (Milkmaids)

Dianella revoluta var. revoluta (Flax-lily)

Dianella tasmanica (Blueberry Flax-lily)

Dichopogon strictus (Chocolate Lily)

Scilla hispanica (Spanish Bluebell) (I)

Wurmbea dioica (Early Nancy)

Wurmbea uniflora (Single-flower Wurmbea)

ORCHIDACEAE

Diuris sulphurea (Tiger Orchid)

Microtis unifolia (Common Onion-orchid)

Pterostylis pedunculata (Maroonhood)

Thelymitra pauciflora (Slender Sun-orchid)

POACEAE

Agrostis aemula (Blown Grass)

Agrostis avenacea (Blown Grass)

Agrostis capillaris (Brown-top Bent) (I)

c c c c

11

$\begin{array}{llllllll}0 & 0 & 1 & 1\end{array}$
Species Zones

D G C P

Aira caryophyllea (Silvery Hair Grass) (I)

Aira elegantissima (Elegant Hair Grass) (I)

Anthoxanthum odoratum (Sweet Vernal Grass) (I)

Briza maxima (Quaking Grass) (I)

Briza minor (Lesser Quaking Grass) (I)

Bromus hordeaceus (Brome) (I)

Cynosurus cristatus (Crested Dog's-tail) (I)

Danthonia caespitosa (Wallaby Grass)

Danthonia dimidiata (Wallaby Grass) (E)

Danthonia pilosa (Wallaby Grass)

Danthonia racemosa (Wallaby Grass)

Danthonia setacea (Wallaby Grass)

Deyeuxia quadriseta (Bent Grass)

Dichelachne rara (Plumegrass)

Ehrharta distichophylla (Ricegrass)

Ehrharta stipoides (Weeping Grass)

Holcus lanatus (Yorkshire Fog) (I)

Paspalum dilatatum (Paspalum) (I)

Pentapogon quadrifidus (Five-awned Speargrass)

Poa annua (Annual Poa) (I)

Poa labillardieri (Tussock Grass)

Poa rodwayi (Tussock Grass)

Stipa mollis (Speargrass)

Stipa pubinodis (Speargrass)

Stipa stuposa (Speargrass)

Themeda triandra (Kangaroo Grass)

Vulpia bromoides (Squirrel-tail Fescue) (I)

POTAMOGETONACEAE

Potamogeton tricarinatus (Floating Pondweed)

$f \circ \quad f$

$f \circ o$

r

o

I

$\begin{array}{lll}r & r\end{array}$

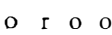

o o

o $\quad$ o

o r

$\begin{array}{llll}0 & 0 & 0 & 0\end{array}$

o r r

r 1

c $f \circ f$

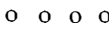

$\begin{array}{lllll}0 & 0 & 0 & 0\end{array}$

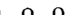

c c c c c

11

$1 \mathrm{r}$

f $\mathrm{o}$ o

$\begin{array}{lllllllllll}0 & o & o & o\end{array}$

f $f$ o f

$\begin{array}{llll}r & r & r & r\end{array}$

1

Typha latifolia (Cats'-tail, Bulrush) (I)

$1 \quad 11$

XANTHORRHOEACEAE

Lomandra longifolia (Sagg)

c c c c

PTERIDOPHYTES

ASPIDIACEAE

Polystichum proliferum (Mother Shield-fern)

ASPLENIACEAE

Asplenium flabellifolium (Necklace Fern)

BLECHNACEAE

Blechnum nudum (Fishbone Warer-fern)

Blechnum wattsii (Hard Water-fern)

DENNSTAEDTIACEAE

Pteridium esculentum (Austral Bracken)

DICKSONIACEAE

Culcita dubia (Common Ground-fern)

Dicksonia antarctica (Soft Tree-fern, Manfern)

HYMENOPHYLLACEAE

Hymenophyllum cupressiforme (Common Filmy-fern)

POLYPODIACEAE

Microsorum diversifolium (Kangaroo-fern)

SINOPTERIDACEAE

Cheilanthes austrotenuifolia (Rock-fern)

$f \quad f \quad f$

f

1

c C C C

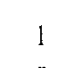

r

1

o

f $f$ o $o$

MOSSES (Only zones are given, no abundances)

\section{AMBLYSTEGIACEAE}

Acrocladium chlamydophyllum

BARTRAMIACEAE

Bartramia ithyphylla

Breutelia affinis

Philonotis tenuis

BRYACEAE

Bryum argenteum

Bryum billardieri

Bryum pacytheca

DICRANACEAE

$\begin{array}{lll}\mathrm{x} & \mathrm{x}\end{array}$

$\begin{array}{llll}x & x & x & x\end{array}$

$\begin{array}{llllllllllllll} & x & x & x\end{array}$

$\mathrm{x}$

$x \quad x \quad x \quad x$

$\mathrm{x}$

$\mathrm{x} \quad \mathrm{x}$ 
Table 1 cont.

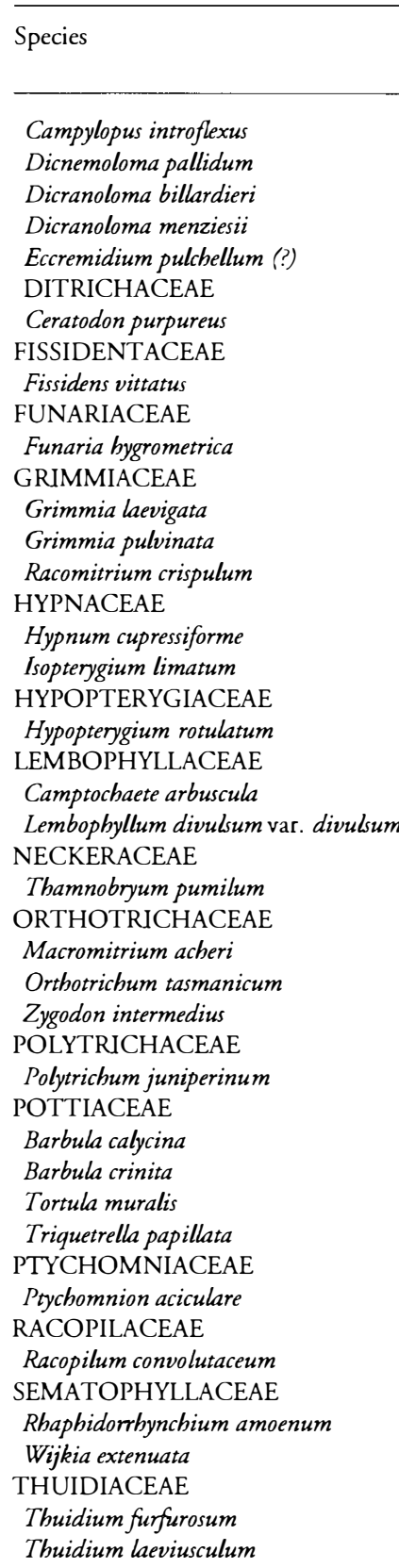

\begin{tabular}{cc}
\hline Species & Zones \\
& D G C P
\end{tabular}

\section{MARCHANTIACEAE}

Lunularia cruciata

METZGERIACEAE

Metzgeria furcata

PLAGIOCHILACEAE

Plagiochila fasciculata

Plagiochila strombifolia

RADULACEAE

Radula buccinifera $\mathrm{x} \times \mathrm{x}$

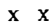

\section{LICHENS (Only species are given, no zones or abundances)}

Arthothelium ilicinum (Taylor) P. James

$\mathbf{x} \quad$ Bacidia cf. millegrana (Taylor) Zahlbr.

$\mathbf{x} \quad$ Buellia disciformis (Fr.) Mudd aggr.

Candelariella xanthostigmoides (Müll. Arg.) R.W. Rogers

$\mathbf{x} \times \mathbf{x} \quad$ Cladia aggregata (Sw.) Nyl.

x Cladia schizopora (Nyl.) Nyl.

Cladonia capitellata (J.D. Hook. \& Taylor) Church. Bab. var. capitellata

$\mathrm{x} \times \quad$ Cladonia corniculata Ahti \& Kashiwadani

Cladonia pyxidata (L.) Hoffm.

$\mathrm{x} \times \quad$ Cladonia ramulosa (With.) Laundon

$\mathrm{x} \times \quad$ Cladonia rigida (J.D. Hook. \& Taylor) Hampe var. rigida Cladonia sulcata Archer var. wilsonii (Archer) Archer

$\mathrm{x} \quad$ Flavoparmelia haysomii (Dodge) Hale

Flavoparmelia rutidota (J.D. Hook. \& Taylor) Hale

$\mathrm{x}$

Graphis cf. desquamescens (Fée) Zahlbr.

Heterodea muelleri (Hampe) $\mathrm{Nyl}$.

$\mathrm{x} \times \mathrm{x} \quad$ Heterodermia obscurata (Nyl.) Trevis.

Hypocenomyce australis Timdal

$\mathrm{x} \quad \mathrm{L} \quad$ Lecideasp. A

Lecidea sp. B

$\begin{array}{llllll}\mathbf{x} & & & \mathbf{x} & \text { Lecidea } \mathrm{sp} . \mathrm{C} \\ \mathbf{x} & \mathbf{x} & \mathbf{x} & \mathbf{x} & \text { Lecidea } \mathrm{sp.} \text { D }\end{array}$

$\mathrm{x} \quad$ ?Lecidea sp.

x $\mathrm{x}$ x Maronea constans Hepp

Menegazzia caesiopruinosa P.James

x $\mathrm{x} \quad$ Menegazzia nothofagi (Zahlbr.) D. Galloway

Menegazzia platytrema (Müll. Arg.) R. Sant.

x $\mathrm{x} \quad$ Menegazzia subpertusa P. James \& D. Galloway

Neofuscelia pulla (Ach.) Esslinger

$\mathrm{x} \times \mathrm{x} \quad$ Parmelia cunninghamii Crombie

$\mathrm{x} \quad$ Parmelia erumpens Kurok.

Parmelia signifera $\mathrm{Nyl}$.

$\mathbf{x} \times \quad$ Parmelina conlabrosa (Hale) Elix \& Johnston

$\mathbf{x} \quad$ Parmelina pseudorelicina (Jatta) Kantvilas \& Elix Parmelinopsis afrorevoluta (Krog \& Swinscow) Elix \& Hale Parmotrema chinense (Osbeck) Hale \& Ahti

Pertusaria gibberosa Müll. Arg.

Phlyctis subuncinata Stirton

Pseudocyphellaria glabra (J.D. Hook. \& Taylor) Dodge

Pseudocyphellaria neglecta (Müll. Arg.) Magnusson

\author{
AYTONIACEAE \\ Asterella drummondii \\ FOSSOMBRONIACEAE \\ Fossombronia sp. \\ FRULLANIACEAE \\ Frullania falciloba \\ Frullania monocera \\ GEOCALYCACEAE \\ Chiloscyphus sp. \\ Chiloscyphus biciliatus (?) \\ Chiloscyphus bispinosus (?) \\ Chiloscyphus semiteres \\ JUNGERMANNIACEAE \\ Jamesoniella colorato \\ LEJEUNEACEAE \\ Lejeunea drummondii \\ Cheilolejeunea mimosa \\ LEPIDOLAENACEAE \\ Gackstroemia weindorferi

$\mathrm{x}$

Punctelia subrudecta (Nyl.) Krog

Pyrrhospora laeta (Stirton) Hafellner

Usnea oncodes Stirton
Ramalina inflata (J.D. Hook. \& Taylor) J.D. Hook. \& Taylor ssp. inflata

Ramalina inflata ssp. australis G.N. Stevens

Rimelia reticulata (Taylor) Hale \& Fletcher

Rinodina sp.

Tephromela atra (Hudson) Hafellner

Thysanothecium scutellatum (Fr.) D. Galloway

Usnea confusa Asahina

Usnea inermis Motyka

$\mathrm{x} \quad$ Usnea rubicunda Stirton

$\mathrm{x} \quad$ Usnea scabrida Taylor ssp. tayloriana G.N. Stevens

Usnea subeciliata (Motyka) Swinscow \& Krog

$\mathrm{x} \times \quad$ Xanthoparmelia flaviscentireagens (Gyelnik) D. Galloway

Xanthoparmelia isidiigera (Müll. Arg.) Elix \& Johnston

$\mathrm{x}$ 